\title{
Investigating karst dissolution loss caused by different intensity droughts using field control experiment
}

\author{
Kaizhong $\mathrm{Li}^{1}$, Yingping Dong ${ }^{1}$, Anning Wang ${ }^{2}$, and Hengsong Wang ${ }^{1}$ \\ ${ }^{1}$ Guizhou Normal University \\ ${ }^{2}$ The people's Government of Anlong County
}

September 21, 2020

\begin{abstract}
Abstract It is well known that droughts have a significant impact on karst dissolution. However, it is not clear how much karst dissolution will be lost under the impact of droughts, especially the loss of different intensity droughts. The present study used a simple but direct standard carbonate tablet to investigated the karst dissolution loss caused by different intensity droughts for four types of land cover in three sites by a field comparative control experiment. Experiment results showed that the drought had caused a significant loss to karst dissolution. Moreover, with the drought increasing, the karst dissolution showed a clear upward trend. From light to rare level, drought caused a 0.4451 to $2.0546 \mathrm{mg} \mathrm{cm}^{-2}$ of a mean loss of karst dissolution for the forest, 0.3044 to $1.9405 \mathrm{mg} \mathrm{cm}^{-2}$ for cropland, 0.5580 to $2.2760 \mathrm{mg} \mathrm{cm}^{-2}$ for shrubland, and 0.5131 to $4.5336 \mathrm{mg} \mathrm{cm}^{-2}$ of a mean loss of karst dissolution for grassland, respectively, which equivalent to a 31.83 to $54.55 \%$ of average loss for the forest, 27.80 to $55.97 \%$ for cropland, 39.97 to $48.03 \%$ for shrubland, and 36.56 to $64.34 \%$ of karst dissolution loss for grassland. Subsequent impact mechanism analysis indicated that soil water plays a crucial role in karst dissolution loss of different intensity droughts. Specifically, drought occurs leads to a decline in precipitation, which in turn undoubtedly reduces the soil water, weakens the karst dissolution process, and finally causes the loss of karst dissolution.
\end{abstract}

\section{KEYWORDS}

Karst dissolution loss, drought, standard carbonate tablets, field control experiment, soil water

\section{Introduction}

Karst dissolution is one of the most fundamental issues in understanding the formation and evolution of karst topography (Ren \& Liu, 1983; Dixon et al., 2001; Phillips, 2005; Ford \& Williams, 2007). At the same time, the karst dissolution process is also an essential part of the global carbon cycle, which plays a pivotal role in global carbon balance and carbon sink estimation (Yuan, 1995; Gombert, 2002; Goudie, \& Viles, 2012; Zeng, Liu, \& Kaufmann, 2019). The key to solving all these problems is to obtain a more realistic amount of karst dissolution.

Understanding and quantifying the influence of different factors on the karst dissolution is the basis of obtaining a proper amount of karst dissolution. Among these factors, the effect of drought on karst dissolution is an important factor that cannot be ignored, especially in global climate change. Observation results indicated that the drought is one of the most frequent natural disasters worldwide (Qiu, 2010; Dai, 2013; Trenberth et al., 2014; Naumann et al., 2018), which has a substantial impact on social economy and the natural environment. Tens of thousands of studies have shown that droughts are becoming more frequent and severe with climate warming (Easterling et al., 2000; IPCC, 2014; Sherwood, \& Fu, 2014). Therefore, investigating the drought loss of karst dissolution has become particularly urgent and vital for karst research. 
The essential characteristic of drought is the deficiency of precipitation, which is the key to the karst dissolution. Some previous studies have observed the effects of droughts on the karst dissolution, mainly caused by precipitation reduction. For example, Zhang (2010) and Wang et al. (2007) found that the karst dissolution in the dry season and rainy season have a noticeable difference. Li et al. (2012) and Lan et al. (2013) also found that the karst dissolution has a more or less decline in the dry season. Nevertheless, the specific amount of karst dissolution loss caused by drought has not been examined in detail, particularly the karst dissolution loss caused by different intensity droughts.

Many methods have developed for karst dissolution estimates and measurements over the past decades (Yuan \& Cai, 1988; Plan, 2005; Ford \& Williams, 2007; Gabrovšek, 2009; Moses, Robinson, \& Barlow, 2014; Kirstein et al., 2016; Pardo-Igúzquiza, Dowd, \& Telbisz, 2020). More popular methods to estimate and measure the karst dissolution are (1) Calculation of solutional denudation by a formula; (2) Weight loss measurement using standard tablets; (3) Short-term lowering of limestone surfaces determined by micrometric measurements; (4) Long-term lowering of bare-rock surfaces determined by measurement of surface irregularities; and (5) Cosmogenic chlorine-36 measurements. Each method has its advantages and disadvantages (Ford \& Williams, 2007; Gabrovšek, 2009; Liu, 2011; Wang, Zhang, \& Qin, 2013), and it might be suitable for different situations. Among these approaches, weight loss measurement using standard carbonate tablets is one of the simplest and most effective ways of estimating the karst dissolution under various environmental conditions (Yuan \& Cai, 1988; Dixon et al., 2001; Plan, 2005). This method is not only the most direct way, but also can be compared with each other, and is widely used in karst research (Yuan \& Cai, 1988; Dixon et al., 2001; Plan, 2005; Zhang, 2010; Hattanji et al., 2014; Krklec et al., 2016).

The karst regions of Southwest China are the most developed karst topography areas, including almost all types of karst landforms in the world. Southwest China has approximately $5.1 \times 105 \mathrm{~km}^{2}$ of karst areas in exposed or outcropped carbonate rock (Jiang, Lian, \& Qin, 2014). It is about 5.8\% of the total land and is distributed mainly in Guizhou and surrounding areas. This area is also a typical ecologically fragile area facing severe ecological and environmental problems (Cai, 1996; Yuan, 1997; Wang, Liu, \& Zhang, 2004; Jiang, Lian, \& Qin, 2014). Because of the particularity of the geological environment and climatic conditions of Southwest China karst regions, karstification is very strong and highly sensitive to changes in the external environment, especially the changes in climate conditions. Unfortunately, drought is also one of the most common natural disasters in central Southwest China (Chen, \& Zhai, 2014; Han et al., 2016), known as "Nine droughts in ten years." Moreover, droughts in this area have an apparent upward trend in recent years (Lin et al., 2015; Liu et al., 2016). As a result, it is of great significance to study the effect of drought on karst dissolution in central Southwest China.

The present study is a first attempt to quantifying the loss impact of drought on karst dissolution and associated impact mechanism using standard carbonate tablets in a field comparative control experiment. This study focuses mainly on the amount of karst dissolution loss caused by different intensity droughts in various areas of central Southwest China. This information is crucial to the study of karst topography, protection of the karst landscape, and the karst carbon balance estimation. At the same time, we will discuss the impact mechanism of drought on karst dissolution loss, which could shed light on studying the karst process in the future.

\section{2 study SITE AND METHODOLOGY}

\subsection{Study site}

Three sites have been chosen for field experiments based on the careful consideration of geological, geomorphic, and climatic conditions in Guizhou Province, central Southwest China (Figure 1). Table 1 shown the main geographic environment information of three experiment sites. Site A (27 $15^{\prime} 37^{\prime \prime N}$, 105deg5'58"E) lies to the Northwest Guizhou, with a relatively cold and dry climate. The topography surrounding the experiment site is a typical karst valley, mostly composed of carbonate rock. Azonal yellow soil covers a large area of Site A, which varied from a few centimeters to approximately one meter in depth. Apart from a thick soil layer in the valley bottom, there is not much soil on steep slopes. The valley has a mixed coniferous 
and broad-leaved forest on the upper part of the hillside, and a few patchy shrub or grass cover underneath. Site B (26deg33'1"N, 106deg19'18"E) is located in a relatively temperate climate region in the central of Guizhou province. A field experiment of Site B was conducted in a giant karst valley. The exposed rock of the valley is relatively pure limestone, which is in contact with a thicker yellow soil above the bedrocks. Compared with Site A, Site B has various vegetation types, mostly consisted of evergreen broad-leaved forest and deciduous broad-leaved forest. The Site C (25deg5'9"N, 105deg19'48"E) is located in a flat karst basin and mainly outcrop rock is dolomitic limestone. As an experiment site in the southwest of Guizhou, which has a humid and warm subtropical climate. The soil around the experiment site is calcareous soil and zonal yellow soil. Due to its suitable climate and soil conditions, the vegetation of these areas is in good condition; most of them are evergreen broad-leaved forest.

These three filed experiment sites are all the typical karst valley or basin landform, and the prominent outcrops are carbonate rocks. The soil and vegetation around these experiment sites are zonal soil and vegetation in Southwest China. From Site A to Site C, the climatic condition gradually changes from a dry and cold climate to a warm and humid environment. The exogenous situation of karst dissolution in three sites is distinct, and it can be regarded as a typical representative of different karst topography in Southwest China.

\subsection{Experiment materials}

To convenience for the comparison of results, the standard carbonate tablets were made of high purity Devonian limestones from Guilin in Guangxi Zhuang autonomous region. This standard carbonate tablet is the most widely used in China. The results of mainly chemical characteristics analysis of sample rocks show that the limestone contains $55.67 \%$ of $\mathrm{CaO}, 0.43 \%$ of $\mathrm{MgO}, 42.96 \%$ of $\mathrm{CO}_{2}$, and $0.66 \%$ acid-insoluble substance, respectively. All limestone rocks were cut and sliced into circular standard-sized tablets, with a diameter of $4.0+-0.1 \mathrm{~cm}$, a thickness of $0.3+-0.02 \mathrm{~cm}$, a surface area of approximately $28.91 \mathrm{~cm}^{2}$, and a weight of $12.45+-0.3 \mathrm{~g}$. Each tablet has two holes in the surface that make the tablet closer to the actual dissolution environment. The bodies of these tablets were all polished.

Before the experiment, the standard tablets are cleaning in distilled water three times and then drying in a laboratory oven for $12 \mathrm{~h}$ at $103 \mathrm{degC}$ and weighing on the accuracy of $0.1 \mathrm{mg}$ by OHAUS analytical balances $(\mathrm{PX} 224 \mathrm{ZH} / \mathrm{E})$. The weighted standard tablets were then labeled and put in a moisture-proof bag for the field experiment. After every experiment period, the tablets retrieved from the field were carefully treated following the same procedure as before the experiment.

\subsection{Field experiment design}

Separating the drought loss of karst dissolution from a normal state is crucial for quantifying the impact of different intensity droughts on karst dissolution. Therefore, the present study concurrently set up two groups of experiments in forest, cropland, shrubland, and grassland of Site A, Site B, and Site C, respectively. In two groups of experiments, one group has no drought treatment, representing the regular karst dissolution. Correspondingly, the other one has drought control treatments, which simulates the karst dissolution affected by droughts. Consequently, the quantity difference between the two groups of experiments can be regarded as the drought loss of karst dissolution.

Transparent rain shelters were established within each experiment plot as the drought treatment during the field experiment. Each treatment was crossing five intensity of droughts (light, moderate, severe, extreme, and rare). Rain shelters are considered ventilation, sunlight, air temperature, rain shelter effectiveness, and so on. To prevent the impact of severe natural drought on karst dissolution, we have prepared an integral emergency response plan based on the multi-year average climate of experimental areas. To the end, of course, none of these measures be used.

The drought intensity levels were determined by the comprehensive consideration of the occurrence of droughts, and the karst dissolution itself in Southwest China. After carefully trade-off, the study used no precipitation in 30 consecutive days as light drought, no precipitation in 60 consecutive days, 90 consecutive 
days, 120 consecutive days, and 365 consecutive days as moderate, severe, extreme, and rare, respectively. Accordingly, the dissolution amounts of obtained after buried 30 consecutive days, 60 consecutive days, 90 consecutive days, 120 consecutive days, and 365 consecutive days in drought control treatments be equivalent to the karst dissolution of affected by light, moderate, severe, extreme, and rare drought, respectively. Correspondingly, the dissolution amounts of obtained after experiment 30 consecutive days, 60 consecutive days, 90 consecutive days, 120 consecutive days, and 365 consecutive days in matched groups can be regarded as the karst dissolution amounts without the effect of light, moderate, severe, extreme, and rare drought, respectively. The quantity difference between the parallel groups in each experiment plot is the karst dissolution loss caused by drought.

\subsection{Data collection}

A total of nine hundred standard tablets were buried in four types of land cover (forest, cropland, shrubland, and grassland) in three experiment sites for different periods. The specific buried pattern of the standard tablet was shown in Figure 2. In forest and cropland, three layers $(-5 \mathrm{~cm},-25 \mathrm{~cm}$, and $-35 \mathrm{~cm}$ of underground) of standard tablets are buried for five experiment periods (30 days, 60 days, 90 days, 120 days, and 365 days), respectively. While in shrubland and grassland, due to the thickness of the soil layer is too thin, only two layers $(-5 \mathrm{~cm}$ and $-25 \mathrm{~cm}$ of underground) of standard tablets were buried. Of the three experiment sites, Site A experimented from August 6, 2017, to August 5, 2018, Site B experimented from August 13, 2017, to August 12, 2018, and Site C experimented from August 1, 2017, to July 31, 2018, respectively. When the burial time reaches 30 days, the first batch of standard tablets shall be taken back for three sites, and so on for the rest of the set of tablets.

To protect the safety of field experiments, we hired local people to manage the experiment sites. After the experiment, 892 out of 900 standard tablets were retrieved from three field experiment sites. Of the eight lost tablets, six of them are broken, and two of them were not found. After the treatment of retrieved tablets, the first data we get is the weight of the standard tablet after the experiment, and the following formula could calculate the karst dissolution amount (Yuan \& Cai, 1988).

$D_{w}=\left(W_{2}-W_{1}\right) \times 1000 \times S^{-1}(1)$

Where $D_{w}$ refers to the karst dissolution amount $\left(\mathrm{mg} \mathrm{cm}^{-2}\right), W_{1}$ is the weight of after experimenting with the tablet $(\mathrm{g}), W_{2}$ is the initial weight of tablet $(\mathrm{g}), S$ is the superficial area of the tablet $\left(\mathrm{cm}^{2}\right)$.

The karst dissolution loss caused by different intensity droughts are calculated:

$D_{L}=D_{\mathrm{wn}}-D_{\mathrm{wt}}(2)$

Where $D_{L}$ is the amount of karst dissolution loss caused by drought $\left(\mathrm{mg} \mathrm{cm}^{-2}\right), D_{\mathrm{wn}}$ is the karst dissolution amount of normal state $\left.(\mathrm{mg} \mathrm{cm})^{-2}\right), D_{\mathrm{wt}}$ is the amount of karst dissolution of drought treatment $\left(\mathrm{mg} \mathrm{cm}^{-2}\right)$.

Removed the broken and missing tablets, each soil layer comprises three tablets in one experiment time. Every dissolution data we obtained from different periods is contained of three or two tablets on average. This method can eliminate some noise of experiment data to a certain extent. Furthermore, it is important to note that the present study is focused mainly on the karst dissolution loss caused by different intensity droughts, rather than accurately measuring the dissolution amounts of karst.

\section{3 results}

The loss of karst dissolution caused by different intensity droughts for four types of land cover in three experimental sites is summarized in Table 2. It is apparent from this data that the drought has caused a great loss to karst dissolution. Under the influence of light drought, the forest has a $0.4451 \mathrm{mg} \mathrm{cm}^{-2}$ of karst dissolution loss (range from 0.0276 to $0.6642 \mathrm{mg} \mathrm{cm}^{-2}$ ), equivalent to $31.83 \%$ of the karst dissolution loss on average. Spatially, Site A has the highest loss, followed by Site B, and then Site C has the lowest loss, which only 5.78 percent of karst dissolution was lost. By comparison, the cropland has experienced a lower loss, has a $0.3044 \mathrm{mg} \mathrm{cm}^{-2}\left(27.80 \%\right.$ ) of karst dissolution loss (range from 0.1695 to $0.4359 \mathrm{mg} \mathrm{cm}^{-2}$ ) due to light drought. Among the three experimental sites, Site C was the most affected area by drought, followed 
by Site B, and Site A was the least affected area. For shrubland, the light drought has caused a $0.5580 \mathrm{mg}$ $\mathrm{cm}^{-2}$ of karst dissolution loss (range from 0.1591 to $0.8094 \mathrm{mg} \mathrm{cm}^{-2}$ ), lost by $39.97 \%$ on average. And the spatial distribution of karst dissolution loss is the same as that of the forest. The karst dissolution loss of light drought for grassland was $0.5131 \mathrm{mg} \mathrm{cm}^{-2}$ in mean value, equal to $36.56 \%$ of the karst dissolution loss. In space, the highest karst dissolution loss of light drought for grassland was Site B, the second-highest loss was Site A, and the third-highest loss was Site C. These data show that the shrubland has the highest karst dissolution loss under the affected of light drought, followed by grassland and forest, and the cropland has the lowest karst dissolution loss in mean value.

Moderate drought can cause an average loss of $37.66 \%, 37.96 \%, 42.06 \%$, and $44.63 \%$ of karst dissolution for the forest, cropland, shrubland, and grassland, respectively. The losses of four kinds of land cover in three experimental sites were clearly higher than that of light drought. Specifically, moderate drought has caused, in mean, a $0.6676 \mathrm{mg} \mathrm{cm}{ }^{-2}$ (range from 0.2871 to $0.9651 \mathrm{mg} \mathrm{cm}^{-2}$ ) of karst dissolution loss for the forest, $0.6203 \mathrm{mg} \mathrm{cm}^{-2}$ (range from 0.2767 to $0.8682 \mathrm{mg} \mathrm{cm}^{-2}$ ) for cropland, $0.8671 \mathrm{mg} \mathrm{cm}^{-2}$ (range from 0.2663 to $1.3317 \mathrm{mg} \mathrm{cm}^{-2}$ ) for shrubland, and $0.9062 \mathrm{mg} \mathrm{cm}^{-2}$ (range from 0.2594 to $1.8125 \mathrm{mg} \mathrm{cm}^{-2}$ ) for grassland, respectively. Qualitatively, the grassland has the highest karst dissolution loss under the impact of moderate drought, followed by shrubland and forest, and the cropland is the least affected land cover types. Regionally, Site B has the highest karst dissolution loss of forest, shrubland, and grassland, followed by Site A, and the Site C is obviously less than that of Site A and Site B. While for cropland, the karst dissolution loss of Site $\mathrm{C}$ was the highest, followed by Site B, and the Site A was the lowest.

The mean losses of karst dissolution caused by severe drought were $0.9247 \mathrm{mg} \mathrm{cm}^{-2}$ (range from 0.4601 to $1.3040 \mathrm{mg} \mathrm{cm}^{-2}$ ) for the forest, $0.8117 \mathrm{mg} \mathrm{cm}^{-2}$ (range from 0.3977 to $1.1034 \mathrm{mg} \mathrm{cm}^{-2}$ ) for cropland, 1.1150 $\mathrm{mg} \mathrm{cm}{ }^{-2}$ (range from 0.2906 to $1.6810 \mathrm{mg} \mathrm{cm}^{-2}$ ) for shrubland, and $1.2372 \mathrm{mg} \mathrm{cm}^{-2}$ (range from 0.2318 to $2.4767 \mathrm{mg} \mathrm{cm}^{-2}$ ) for grassland, respectively. The loss percentage of four kinds of land cover was over $40 \%$, ranged from $42.80 \%$ to $46.80 \%$ on average. The karst dissolution loss caused by severe drought, in mean, from high to low was grassland, shrubland, forest, and cropland. By region, the highest dissolution loss of forest occurred in Site B, then Site A, and finally the Site C. To cropland, Site C is the most hit by severe drought, followed by Site B, and the Site A hit by drought lowest. Affected by severe drought, the shrubland of Site A has the highest karst dissolution loss, followed by Site B, and Site C has the lowest loss. About grassland, the regional loss pattern is the same as the forest.

As shown in Table 2, once extreme drought occurs, the forest will suffered a $0.8602 \mathrm{mg} \mathrm{cm}^{-2}$ (range from 0.5742 to $1.1830 \mathrm{mg} \mathrm{cm}^{-2}$ ) of karst dissolution loss, $0.9316 \mathrm{mg} \mathrm{cm}^{-2}$ (range from 0.5638 to $1.2349 \mathrm{mg} \mathrm{cm}^{-2}$ ) of loss for cropland, $1.1668 \mathrm{mg} \mathrm{cm}^{-2}$ (range from 0.3770 to $1.8817 \mathrm{mg} \mathrm{cm}^{-2}$ ) of loss for shrubland, and $1.6108 \mathrm{mg}$ $\mathrm{cm}^{-2}$ (range from 0.2421 to $3.0682 \mathrm{mg} \mathrm{cm}^{-2}$ ) of loss for grassland, respectively, were lost by $42.00 \%, 43.27 \%$, $45.19 \%$, and $50.36 \%$, on average. The mean loss of karst dissolution to grassland was the highest, followed by shrubland and cropland, and the forest has the lowest karst dissolution loss in amount and rate. Like severe drought, the spatial loss patterns of karst dissolution caused by extreme drought for four kinds of land cover types are the same as severe drought. From these data, we found an interesting phenomenon. The extreme drought caused the loss of karst dissolution of forest in Site A and Site B, as well as the karst dissolution loss of shrubland in Site B, which is slightly lower than that of severe drought. Besides, although the karst dissolution loss of grassland in Site C caused by extreme drought does not drop in amount compared to the severe drought, it is lower than that of loss of moderate drought. These results suggest that the karst process may be deposited under the influence of extreme drought. Of course, this does not rule out the effect of abnormal experimental data.

Not surprisingly, rare drought has caused the highest loss to karst dissolution. On average, rare drought has caused a $2.0546 \mathrm{mg} \mathrm{cm}^{-2}$ (range from 0.9616 to $2.6323 \mathrm{mg} \mathrm{cm}^{-2}$ ) of karst dissolution loss for the forest, $1.9405 \mathrm{mg} \mathrm{cm}^{-2}$ (range from 1.0065 to $2.7327 \mathrm{mg} \mathrm{cm}^{-2}$ ) for cropland, $2.2760 \mathrm{mg} \mathrm{cm}^{-2}$ (range from 0.5258 to $3.3794 \mathrm{mg} \mathrm{cm}^{-2}$ ) for shrubland, and $4.5336 \mathrm{mg} \mathrm{cm}^{-2}$ (range from 0.5500 to $8.3984 \mathrm{mg} \mathrm{cm}^{-2}$ ) for grassland, which equivalent to $54.55 \%$ of karst dissolution loss for the forest, $55.97 \%$ of loss for cropland, $48.03 \%$ of loss for shrubland, and $64.34 \%$ of loss for grassland, respectively. In total, grassland suffered the highest karst 
dissolution loss under the impact of rare drought, followed by shrubland and forest, and then the cropland has the lowest loss of karst dissolution. In terms of spatial loss, rare drought has the greatest impact on forest and shrubland in Site A, followed by Site B, and the forest and shrubland of Site C have the least effects. While for cropland, Site B has the highest loss of karst dissolution, followed by Site C, and Site A has the lowest loss of karst dissolution. For grassland, Site B suffered the biggest loss of karst dissolution, followed by Site A, and Site C suffered the lowest loss of karst dissolution.

The vertical analysis showed that there had been a clear increase trend in karst dissolution loss with the rise of drought intensity (Figure 3, Figure 4). With the drought intensity increased from light to severe, the karst dissolution losses of almost all land cover types in amount were increased except for the grassland in Site C (Figure 3). When drought intensity rises to the extreme, the dissolution loss of forest in Site A and Site B, and the dissolution loss of shrubland in Site B have a slight drop. The karst dissolution loss of all types of land cover in three experimental sites was increased along with the strengthen of drought intensity. Although the loss percentage of karst dissolution is not consistent with the increase of drought intensity, it also shows a clear upward trend (Figure 4). Regionally, almost all karst dissolution loss of land cover in Site $\mathrm{C}$ was clearly lower than Site A and Site B except the cropland. According to the experimental results, the lower amount of karst dissolution loss does not mean that the loss rate is small such as the karst dissolution loss of forest in Site C. It also shows that the influence of drought on karst dissolution loss in different areas is exceptionally related to karst environment.

\section{Discussion}

\subsection{Impactmechanism ofdroughts on karst dissolution}

Karst dissolution is essentially the physical and chemical action of water on soluble rocks (Ren \& Liu, 1983; Ford \& Williams, 2007). In other words, water is the most crucial factor in karst dissolution. Critically, the water in karst areas mainly comes from atmospheric precipitation, particularly in karst areas of Southwest China. That is the key to the effect of drought on karst dissolution. Theoretically, drought leads to a decline of precipitation and, in turn, reduces the soil water, weakens the karst dissolution process, and finally causes the loss of karst dissolution. That is the primary mechanism of the impact of drought on karst dissolution.

To better understand the impact mechanism, three factors, soil water, soil $\mathrm{pH}$, and soil organic matter, which are most closely related to karst dissolution, were selected to study the influence of drought on karst dissolution. Among the three factors, soil water is the most critical prerequisite for karst dissolution and is also the carrier of $\mathrm{CO}_{2}$ forming carbonic acid in the soil. Soil carbonic acid is a direct cause of karst dissolution, which is generally measured by soil $\mathrm{pH}$ value. In theory, the more soil water, the lower the soil $\mathrm{pH}$, and then the higher the karst dissolution, and vice versa. In contrast, soil organic matter is an indicator of the soil environment, which significantly impacts soil water and soil $\mathrm{pH}$ levels. For example, high soil organic matter means that the soil structure is better and could maintain more water and $\mathrm{CO}_{2}$ in the soil. Naturally, more soil carbonic acid can be formed. There is no doubt that many other factors can affect karst dissolution, but they are mainly achieved by soil water and soil carbonic acid. And absolutely, it is of great significance to consider the influence of other factors in future research.

Data collection and field experiments were carried out simultaneously. Moreover, each data is composed of no less than three samples. In the experiment, soil water was measured by a portable soil water meter of Spectrum (TDR 300, accuracy is $0.1 \%$ in Volume content). Soil pH was investigated by a portable soil acidity meter (FieldScout $\mathrm{pH} 400$ ) with an accuracy of +-0.01 in value. Soil organic matter was analyzed by potassium dichromate sulfuric acid solution titration in a laboratory. The results show that the soil water decreased obviously under the influence of drought, while soil $\mathrm{pH}$ showed an upward trend (Figure 5). Interestingly, there is no apparent change trend of soil organic matter under the influence of drought except in Site A (Figure 5). These results suggest that drought directly affected soil water and soil $\mathrm{pH}$, but the impact on soil organic matter was not clear.

To further understand the mechanism, we applied a Pearson correlation analysis to research the correlation between karst dissolution loss and soil water, soil $\mathrm{pH}$, and soil organic matter. To exclude the influence of 
time, season, and other factors on soil water, soil $\mathrm{pH}$, and soil organic matter, all the data used for analysis were from the drought control group. As expected, there was a clear negative correlation between karst dissolution loss and soil water, but a positive correlation between karst dissolution loss and soil $\mathrm{pH}$ (Table 3). Meanwhile, there has a negative correlation between soil water and soil $\mathrm{pH}$ (Table 3). Besides, there is no consistent correlation between karst dissolution loss and soil organic matter or soil organic matter and soil water and soil pH, except for Site A (Table 3). The results show that soil water plays a crucial role in the karst dissolution process; soil carbonic acid results from the interaction between soil water and soil environment. Finally, the effect of soil organic matter on karst dissolution is uncertain.

Although only three factors are used in our study, the results clearly described the critical influence process of drought on karst dissolution. Simultaneously, we also noticed that continuous field observation is crucial to understand the effect of drought on the karst dissolution process. Whatever the outcome, this experiment had demonstrated that soil water was the center of the impact of drought on karst dissolution, and the soil carbonic acid is an indirect control factor. As for soil organic matter, its effect is still uncertain, but in most cases, increasing soil organic matter is beneficial to mitigate the impact of drought on karst dissolution.

\subsection{Drought loss resistance of different land covers}

It is generally believed that higher land coverage might have led to a lower drought loss of karst dissolution. That means, in our study, the forest should have the lowest drought loss of karst dissolution, followed by shrubland and grassland, and then cropland should have the highest drought loss. However, the drought loss of karst dissolution for four types of land cover does not follow this law, especially the Site C. The results indicated that the land coverage is not the only reason for the drought loss resistance of karst dissolution. It should be the integrated result of multiple factors.

In principle, the higher the drought loss resistance, the lower the karst dissolution loss. Based on this principle, the study ranked the drought loss resistance of karst dissolution for four land cover types and shown in Figure 6. Totally, cropland in Site A has the highest drought loss resistance of karst dissolution, followed by forest and grassland, and shrubland has the lowest drought loss resistance of karst dissolution (Figure 6). The drought loss resistance of karst dissolution in Site B is similar to Site A, but the lowest drought loss resistance of karst dissolution is grassland (Figure 6). Surprisingly, the highest drought loss resistance of karst dissolution in Site C is grassland, followed by shrubland and forest, and cropland has the lowest drought loss resistance of karst dissolution (Figure 6).

As mentioned above, the drought loss resistance of karst dissolution is not only related to land coverage, but also the other factors. According to the data we measured (Table 4), soil pH may be, at least partly, the cause of the high drought loss of shrubland and grassland in Site A and Site B, while the drought loss resistance of karst dissolution in Site C may be related to soil organic matter. It must be noted that the available data can not completely clarify the actual reason for the drought loss difference of different land cover types. Nevertheless, a relatively straightforward qualitative result has an unmeasurable application for karst research and landscape protection, especially when considering afforestation to deal with climate change.

\subsection{Response of karst dissolution to drought in different areas}

A striking result of the study is that the drought losses of karst dissolution of Site C were sharply lower than Site A and Site B except for cropland. However, in terms of the proportion of losses, the drought losses of Site $\mathrm{C}$ are not as small as seems (Table 2). Small karst dissolution loss in Site C may be directly related to the lower natural karst dissolution of Site C. It has long been assumed that the wetter and warmer the area is, the higher the karst dissolution should be. However, the karst dissolution in Site $\mathrm{C}$ with wetter and warmer conditions is less than that in Site A and Site B. More than that, the karst dissolution loss of cropland in Site A and Site B is the smallest, while that in Site C cropland becomes the most loss of land cover type.

According to the climate data recorded at Anlong station, which nearly by Site C (Table 5), during the 
experiment period, there is no severe drought occurred in Site C. Within experiment time, there was only a slight increase in precipitation, while the temperature increased by one . Simultaneously, the largest continuous rainless days are 12 days, and even the largest continuous no effective precipitation days are only 21 days. In other words, during the study period, the climate of Site $\mathrm{C}$ has not changed abnormally. So, climate change should not be the main factor of the lower karst dissolution loss for Site C.

Besides, we analyzed the soil environment carefully, and we found that the soil $\mathrm{pH}$ value of Site $\mathrm{C}$ was lower than that of other regions in total (Tables 4). Furthermore, except for the slightly higher soil organic matter in Site C, there is not much difference in the three sites (Tables 4). According to our data, it is difficult to determine the reason for the lower karst dissolution of Site C. Of course, in other studies, there are also cases where the dissolution environment is pleasant, but the karst dissolution is not as high as expected (Yuan \& Cai, 1988). These results suggest that the karst dissolution results from the combined effect of geology, soil, climate, and vegetation. Additionally, it may also relate to the specific experiment site and time.

\subsection{Results comparison and validation}

Unlike the loss of drought in the economic and social fields, few records are about the loss of karst dissolution. On the other hand, most previous studies pay a little attention to the drought loss of karst dissolution due to different purposes. Therefore, it is quite challenging to compare the results of such studies, especially based on the same experiment material. Nevertheless, we obtained the 365 days of natural karst dissolution can be used for results comparison and validation. At least it can increase the understanding of our experimental methods and results.

The most formal and credible karst dissolution data was published by Yuan \& Cai in 1988 (Table 6). Moreover, Yuan \& Cai (1988) use the same materials and methods as ours. They carried out experiments on three layers of ground, underground $20 \mathrm{~cm}$ and underground $50 \mathrm{~cm}$ (Yuan \& Cai, 1988), while our results were from the underground $5 \mathrm{~cm}, 20 \mathrm{~cm}$, and $50 \mathrm{~cm}$. The difference is that Yuan \& Cai (1988) only launched one experiment in each area, while we did four types of the land cover of karst dissolution experiments. For easy comparison, we summarized the dissolution data of the sites near the study area and shown in Table 6 . The results of this study are shown in Table 7 .

On average, karst dissolution of Yuan \& Cai (1988) is range from 0.5803 to $7.4511 \mathrm{mg} \mathrm{cm}^{-2}$ in one year (Table 6), and our dissolution data is range from 1.4251 to $9.7855 \mathrm{mg} \mathrm{cm}^{-2}$ (Table 7), which is slightly higher than the data of Yuan \& Cai (1988). We then calculated the average karst dissolution amounts of four land types in each experiment site, ranging from 2.1783 to $6.2011 \mathrm{mg} \mathrm{cm}^{-2} \mathrm{a}^{-1}$ (Table 7). This data is close to the result of Yuan \& Cai (1988). A detailed comparison results show that the dissolution data of the present study is higher than Guangzhou and lower than Guilin on average value under similar conditions (Table 6, Table 7). Among the six experimental sites of Yuan \& Cai (1988), Guiyang is very close to Site B, but the karst dissolution is lower than our result (Table 6, Table 7). Of course, the reason for the difference in results is not apparent.

In 2010, Zhang (2010) conducted a similar experimental research, obtained a $0.1117 \mathrm{mg} \mathrm{cm}^{-2} \mathrm{a}^{-1}$ of dissolution rate for the cropland, $5.0031 \mathrm{mg} \mathrm{cm}{ }^{-2} \mathrm{a}^{-1}$ for forest, $0.7617 \mathrm{mg} \mathrm{cm}^{-2} \mathrm{a}^{-1}$ for shrub grass, and $4.3407 \mathrm{mg} \mathrm{cm}^{-2}$ $\mathrm{a}^{-1}$ for grassland on average, respectively. Among them, the karst dissolution of cropland and shrub grass is less than our results, and the rest are proximity to us (Table 2, Table 7). Some kindred studies have been conducted to test the karst dissolution, and the results have resembled Zhang. Almost certainly, these results indicated that there is some difference between our results to the formerly studies, but there was a notable difference was found. Furthermore, each of our dissolution data has three parallel data, and there should be a high degree of credibility.

\section{Conclusions}

It is well known that drought has a significant impact on karst dissolution. However, it is not clear how much karst dissolution will be lost under the influence of different intensity droughts. With the help of field control comparative experiments, we provide relatively detailed results for the first time to answer the question. 
We clearly know the karst dissolution loss caused by different intensity droughts for different land types and in different regions through the investigation. Although there may be some limitations in the study, the experiment results confirmed that the drought has a considerable impact on karst dissolution from the quantity point of view.

Controls of mechanism analysis suggest that the effect of drought on karst dissolution is mainly achieved by reducing soil water. Specifically, drought leads to a decrease of precipitation and, in turn, reduces the soil water, weakens the karst dissolution process, and finally causes the loss of karst dissolution. This results very clearly implies an essential role of precipitation and soil water on the karst dissolution. Previous studies have also demonstrated this argument.

\section{Acknowledgements}

This research was funded by the National Natural Science Foundation of China $(41561047,41561064)$, Doctoral Foundation of Guizhou Normal University (No. 2012), and the Guizhou Science and Technology Supporting Plan (No. 2016-2841).

\section{Data availability statement}

The data that support the findings of this study are available from the corresponding author upon reasonable request.

\section{Hosted file}

Figures of manuscript.docx available at https://authorea.com/users/360099/articles/481816investigating-karst-dissolution-loss-caused-by-different-intensity-droughts-using-fieldcontrol-experiment

\section{Hosted file}

Tables of manuscript.docx available at https://authorea.com/users/360099/articles/481816investigating-karst-dissolution-loss-caused-by-different-intensity-droughts-using-fieldcontrol-experiment 